\title{
Homodyne OFDM with Optical Injection Locking for Carrier Recovery
}

\author{
Zhixin Liu, Member, IEEE, Joonyoung Kim, David S. Wu, David J. Richardson, Member, IEEE, and \\ Radan Slavík, Senior Member, IEEE
}

\begin{abstract}
Homodyne detection provides the simplest digital signal processing (DSP) solution to optical coherent detection and minimizes the receiver bandwidth requirements. These features make it promising for high spectrally-efficient formats such as Optical Orthogonal Frequency Domain Multiplexing (OFDM), which has a flat optical spectrum and which is thus inherently sensitive to high frequency distortions, e.g., due to limited detector bandwidth. The key to homodyne detection is recovery of the carrier from the received signal all optically (as opposed to frequency offset compensation via digital signal processing. Herein we use optical injection locking (OIL) in conjunction with carrier tone-assisted OFDM to achieve this. In contrast to previous reports, we show that OIL carrier recovery with subsequent homodyne detection can operate without the need for any optical pre-filtering. First, we evaluate the performance as a function of the carrier tone guardband bandwidth. Further, we improve the robustness of this technique using a slow phase lock loop that compensates for drift in the laser's temperature/current control electronics. Using this improved setup, we compare our all-optical-carrier-recovered homodyne and the 'traditional' DSP-assisted intradyne detection for the case of OFDM-16QAM signals. Finally, we compare the computing complexity necessary for both approaches and estimate the intradyne performance limitations due to the carrier-local oscillator frequency offset.
\end{abstract}

Index Terms - Optical fiber communication, homodyne detetion, OFDM, optical injection locking.

\section{INTRODUCTION}

$\mathrm{M}$ ODERN optical communication systems rely on encoding data using multiple amplitude and phase levels to increase the spectral efficiency of transmission. Orthogonal Frequency Division Multiplexing (OFDM) represents a promising signaling technique because it is efficient in mitigating inter-symbol interference (ISI) caused by chromatic dispersion (CD) and polarization mode dispersion (PMD) [1]. Its further attraction is that it allows software-defined bandwidth allocation and it is therefore considered an enabling technology for flexible high-speed optical networks [2]. Despite these advantages, its high sensitivity to carrier frequency offset (CFO) and phase noise remain issues for any practical implementation. Thus, extensive research has been carried out on estimating and compensating the CFO and phase

The authors are with the Optoelectronics Research Centre, University of Southampton, Southampton, United Kingdom, SO17 1BJ (e-mail: z.liu@soton.ac.uk).

This research has been supported by EPSRC Fellowship grant agreement no. EP/K003038/1 and EPSRC grant Photonics Hyperhighway, no. EP/I061196X. noise through digital signal processing (DSP) [3]. Although DSP was proven to successfully provide such compensation, residual CFO may still degrade the performance and it requires additional computational resource [4].

Homodyne detection is in principle the most straightforward solution to coherent detection. It has the lowest DSP requirements (i.e. no CFO compensation and straightforward carrier phase recovery), and the lowest optoelectronic and electronic bandwidth requirements. This latter point is particularly important for high spectral efficiency formats such as OFDM which have a flat spectrum [5], i.e., which carry a significant portion of the information at high frequencies and hence suffer from the detection-limited bandwidth, especially when the CFO is large [6]. However, homodyne detection requires the optical carrier to be recovered prior to coherent detection (i.e. in the optical domain). In our opinion, to be practical, any homodyne implementation should rely on the same or at least similar optical hardware as the DSP-based intradyne receivers used today.

Optical carrier recovery techniques can be classified into two categories: (i) optical phase lock loop (OPLL) based and (ii) optical injection locking (OIL) based. OPLL based approaches have been studied for many years and significant recent progress has been made with the advent of photonic integrated circuits $[7,8]$. The alternative OIL technique is conceptually simple and capable of tracking the carrier frequency over a wide range, allowing the use of a large-linewidth (and thus low-cost) local oscillator (LO) in coherent detection. Promising as it looks, the technique does require the presence of an optical carrier tone in the signal to which the LO can be locked. Fortunately, a carrier tone is usually inserted in the middle of the OFDM spectrum (carrier-aided OFDM). Although this requires the IQ modulator to be biased slightly away from its optimum point resulting in a slight increase in the nonlinearity of the modulator response [9], it proves to be beneficial at the receiver side for DSP-based CFO estimation, phase noise estimation, and fiber nonlinearity compensation [10,11]. Moreover, a guardband that isolates the carrier tone from the data can be easily created by setting the low frequency OFDM sub-carriers to zero. All these features make OFDM a promising format for OIL-based homodyne detection (as first suggested in [12]), especially when compared to single-carrier modulation formats, in which carrier tone insertion is far less straightforward [13-17]. Although promising and elegant, the early proof-of-principle demonstration [12] required a large protection guardband (reducing transmission capacity by $\sim 20-30 \%$ ) and a narrow band optical filter for carrier tone 
pre-filtering before the OIL, substantially compromising the attraction of this method for practical application.

Recently, we proposed a modification of this OIL technique [18], simultaneously mitigating its two main drawbacks (severe reduction of transmission capacity and need for an ultra-narrow optical filter prior to OIL). We demonstrated that when the locking range is small enough, the optical carrier can be cleanly recovered by direct injection (i.e. without any optical pre-filtering) OIL of a semiconductor laser with almost-negligible reduction of the bandwidth to be sacrificed due to the carrier tone protection guardband. We also showed that OIL improved the linewidth of the LO, allowing us to obtain lower phase noise and better performance at no extra cost.

In this paper, we expand our previous research with additional studies and comparisons. We first study the minimum required guardband bandwidth by reducing the locking range and compare its performance with different detection schemes. To enable robust operation even at very narrow locking ranges (for which any significant drift in the lasers' driving electronic can cause locking to be lost), we implemented a slow (and thus also low-power and low-cost) feedback control circuit to track the signal carrier. With this improved system, a more complex modulation format than-previously-reported (16 QAM) was tested for subcarrier constellation mapping, demonstrating scalability of this approach to higher order modulation formats. In all of our experiments, the performance of the OIL-based homodyne detection was compared with that of the mainstream DSP-based intradyne detection used today.

The principle of our OIL-based carrier recovery and the pilot-aided phase lock loop is introduced in Section II. The setup of our experiment and the different receiver architectures investigated are described in Section III. Our results are shown and discussed in sections IV and V, respectively.

\section{INJECTION-LOCKING FOR OPTICAL CARRIER RECOVERY}

\section{A. Principle of the OIL-based Carrier Recovery}

The principle of OIL-based carrier recovery is shown schematically in Fig. 1. In carrier-aided OFDM (Fig.1 (b)), several subcarriers around the center carrier tone are set to zero to form a guardband which ensures clear carrier tone extraction for the DSP-based, or OIL-based, phase estimation. The OIL process can be viewed as a narrow band filter that filters out any signal at frequencies beyond the OIL bandwidth. By injecting the carrier-aided OFDM signal into a slave laser, the injection locking process not only filters the data signal, but also amplifies the injected carrier tone, which makes it a perfect candidate for optical carrier recovery [19]. The key is to set the injection locking range to be smaller than the guardband bandwidth. Indeed, a smaller guardband leaves more bandwidth for the data, resulting in a higher transmission capacity. Thus, intuitively, the smaller the injection locking range the better.

The locking range of a semiconductor laser for continuous wave (CW) injection can be expressed as [20]

$$
\Delta \omega_{L}=f_{d} \sqrt{\frac{P_{i}}{P_{o}}} \cdot \sqrt{1+\alpha^{2}}
$$

where $f_{d}$ is the slave laser cavity mode spacing, $P_{i}$ the CW injected power, and $P_{o}$ the output power of the slave laser. $\alpha$ is known as the linewidth enhancement factor which accounts for the phase-to-amplitude optical field coupling inside the laser cavity. As we can see from (1), for a given laser ( $f_{d}$ and $\alpha$ fixed by the slave laser geometry and active medium properties) the locking range is proportional to the square root of the injection ratio $P_{i} / P_{o}$. For a given slave laser output power, a weak injection power is needed to obtain a narrow locking range.

The effective OIL filtering profile is shown as a dotted line in Fig. 2(a). At the edge of the locking range, the filter profile is very sharp, being inversely proportional to the square of the frequency deviation between the inserted carrier and the free-running slave laser $\left(\propto 1 / \Delta \omega^{2}\right)$ [19].

a
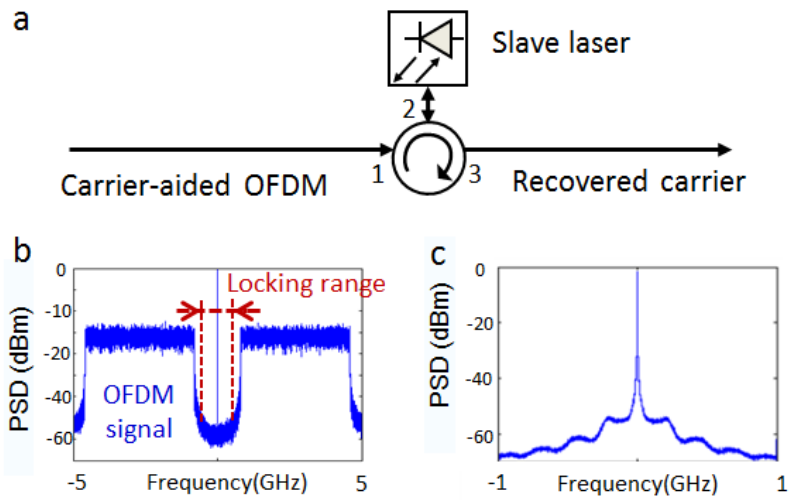

Fig. 1. OIL-based carrier recovery: a) schematic, b) OFDM signal with a carrier tone that is separated from the data using a guardband. For the OIL-based carrier recovery, the OIL locking range must be smaller than the guardband width; c) recovered carrier.

\section{B. Stabilization of the Carrier Recovery with Pilot-aided Phase Lock Loop}

In a real system, both the transmitter laser and the LO carrier frequencies drift, mainly due to the drift of their drive electronics (temperature and current controllers). To keep a stable narrow OIL locking range the center wavelength of the LO must be controlled to track that of the transmitter laser. In our work, we used a modification of a previously-developed tracking technique in which stable injection locking of a slave laser was achieved using a slow (sub-kHz) phase locked loop (PLL) [21].

The principle of how the error signal for our PLL is generated is illustrated in Fig.2. The carrier tone - being within the injection locking range - experiences a phase change that depends on its position within the locking range given by $\Delta \phi=-\arcsin \left(\Delta \omega / \Delta \omega_{L}\right)-\arctan \alpha$, where $\Delta \phi$ is the difference between the phase of the master signal incident to the slave laser and the optically-injection-locked slave output [20]. Thus, $\Delta \omega$ (difference in the free-running frequencies of the master signal and slave laser, which we need to keep close-to-zero) can be found by measuring $\Delta \phi$. To find $\Delta \phi$, we inserted a carrier pilot tone into the guardband of the carrier-aided OFDM signal 
(Fig.2 (a)). This pilot tone, carrier tone, and OFDM signal are all generated together in the digital domain, just before the digital-to-analog convertor (DAC), without the need for any additional components. The OIL bandwidth is set to be narrower than the pilot tone frequency, causing the pilot tone sidebands to be outside of the locking range and they therefore do not experience the phase shift $\Delta \phi$. Consequently, information about $\Delta \phi$ (and thus $\Delta \omega$ ) can be extracted by measuring the phase of the beat between the injection-locked carrier and the pilot tone side-bands, all of them being conveniently present at the slave laser output, Fig. 2(b). After being detected by a photodetector, the radio frequency (RF) beat signal between the carrier and the pilot tone is given by:

$$
I=1+\frac{\eta^{2}}{2}+\frac{\eta^{2}}{2} \cos \left(2 \omega_{p} t\right)+2 \eta \cos \left(\omega_{p} t\right) \cos (\Delta \phi)
$$

where $\eta$ is the normalized intensity ratio between carrier and pilot tone, and $\omega_{p}$ is the angular frequency of the pilot tone. The third term of (2) carries the desired information on $\Delta \phi$, which is used as an error signal for the PLL after being down-converted to the baseband using an RF mixer.

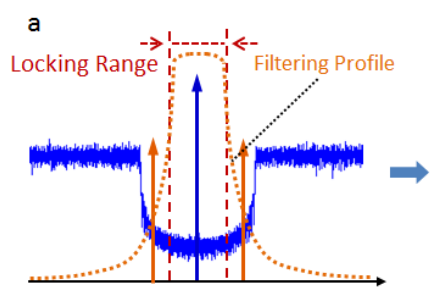

Injected signal

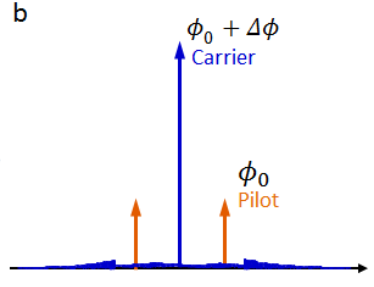

Recovered carrier
Fig. 2. Error signal generation using a pilot tone: a) A pilot tone applied to the carrier generates sidebands that need to be outside the OIL locking range, but within the pilot tone guardband (to prevent interference with the data); b) At the slave laser output, the carrier acquires a phase shift with respect to the pilot tones, which is retrieved and used as the phase lock loop error signal.

\section{EXPERIMENTAL SETUP}

Fig. 3 shows our experimental setup. The waveform samples of the base band OFDM are calculated offline based on a pseudorandom bit sequence (PRBS) of $2^{17}-1$ length. QPSK and 16QAM were used for subcarrier constellation mapping. The electrical waveforms were generated by an arbitrary waveform generator (AWG) operating at $10 \mathrm{GS} / \mathrm{s}$ which then drove an I-Q modulator. An IFFT (Inverse Fast Fourier Transform) size of 256 was used with 11 high frequency subcarriers set to zero. An eight-sample cyclic prefix (CP) is placed before and after each OFDM symbol, resulting in a symbol rate of about 36.8 $\mathrm{MSym} / \mathrm{s}$ for each subcarrier. A clipping ratio factor of 3.6 (i.e. with a clipping boundary equal to 3.6 times the standard deviation of the original waveform) was implemented through digital signal processing. To investigate the sensitivity of the OIL-based carrier recovery scheme to the guardband width we generate OFDM signals with a carrier guardband occupying 2 , 6,14 , or 20 sub-carriers (by setting them to zero).

A laser with $10 \mathrm{kHz}$ linewidth emitting at $1557.4 \mathrm{~nm}$ was used in the transmitter. It is worth mentioning that our choice of the transmitter laser was not made based on its narrow linewidth, as OIL's inherently-large bandwidth allows for tracking significantly broader linewidth lasers (e.g., $>1 \mathrm{MHz}$ [22]). The output power of the laser was $13 \mathrm{dBm}$. Prior to the data modulation, a 50/50 splitter taps off half of the CW light, which is then used for homodyne detection for comparative purposes (details are given later). The I-Q modulator was biased slightly away (about $1.03 \mathrm{~V}_{\pi}$ ) from the transmission null point for both I and Q quadratures to generate the carrier tone. To ensure good linearity, $V_{p p}$ (peak-to-peak) of the RF drive signals was reduced to $\sim 0.6 V_{\pi}$. The carrier-to-signal power ratio (CSR) was $-9 \mathrm{~dB}$.

The transmission link consisted of two distinct fiber spans (75 km standard single mode fiber (SSMF-28) and $80 \mathrm{~km}$ large effective area fiber (LEAF)) providing a total transmission distance of $155 \mathrm{~km}$. This fiber was chosen simply based on availability rather than based on any particular design criteria. The optical power launched into the transmission link was -2 $\mathrm{dBm}$.

At the receiver side, ASE-generated noise loading was used to adjust the optical signal-to-noise ratio (OSNR). The single-polarization OSNR after two-span transmission was 34 $\mathrm{dB}$. To study the performance of our carrier recovery scheme and to compare it with conventional homodyne and intradyne schemes, different receiver front-end set ups (described in detail later in this section) were used prior to coherent detection. After coherent detection, the electrical signals were sampled by an $80-G S / s$ real time oscilloscope before offline processing.

In the offline processing, an 8\% training overhead was used for symbol synchronization and channel estimation. The common phase error (CPE) was estimated from the OFDM center tone after digitally filtering with a $35-\mathrm{MHz} 3-\mathrm{dB}$

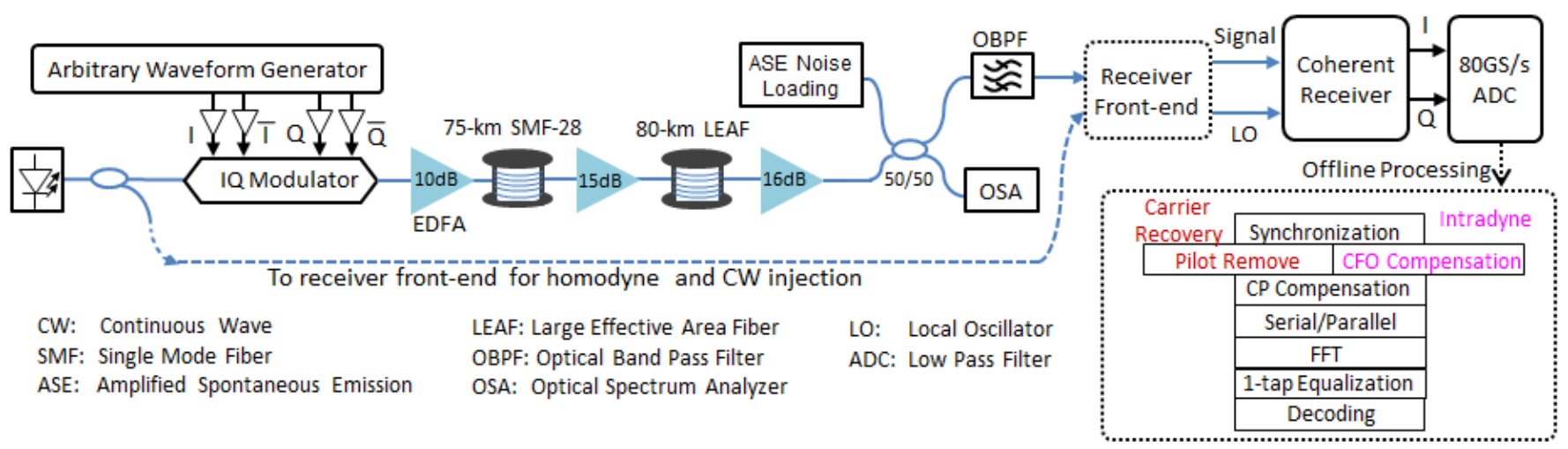

Fig. 3. Setup of the transmission experiment. 
bandwidth $5^{\text {th }}$ order Bessel-profile low-pass filter. For intradyne detection, the CFO was estimated by calculating the frequency offset of the carrier tone at a resolution of $200 \mathrm{kHz}$ and this was then compensated by shifting the signal back to the baseband, both estimation and compensation performed directly in the frequency domain. Finally, the bit error ratio (BER) was calculated after 1-tap equalization and decoding. a

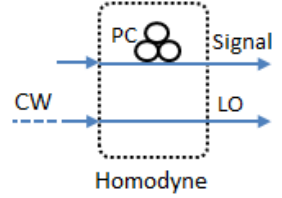

C

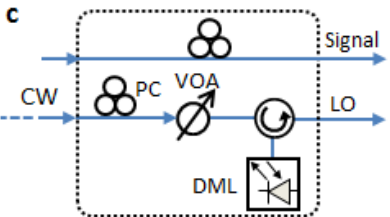

Carrier recovery using $\mathrm{CW}$ injection

e

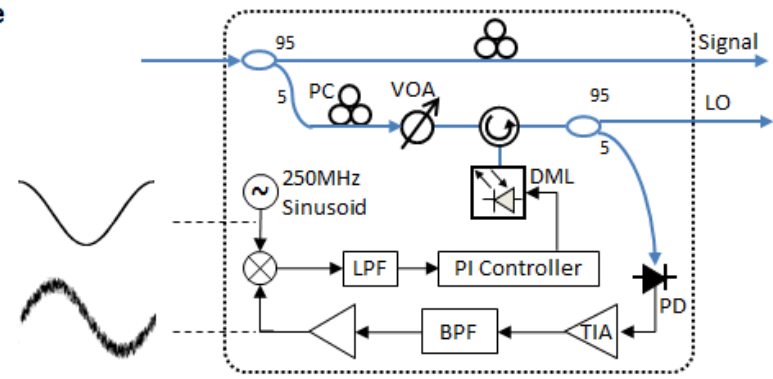

OIL-based carrier recovery with feedback control

VOA: Variable Optical Attenuator DML: Directly-modulated Laser LPF: Low Pass Filter BPF: Band Pass Filter

TIA: Transimpedance Amplifier PD: Photon Detector PC: Polarization Controller PI: Proportional-integral
Fig. 4. Setup of the different receiver front-ends: (a) homodyne detection; (b) intradyne detection; (c) OIL-based carrier recovery using CW injection; (d) OIL-based carrier recovery enabled homodyne detection; (e) set-up from (d) complemented with a slow phase-locked loop.

The different receiver front-end setups are shown in Fig. 4. A 0.12-nm OBPF was used to reject optical noise in setups shown in Fig. 4 (a)-(d). This narrow bandwidth filter allowed us to filter out most of the out-of-band noise already present in the optical domain. The first two (Fig. 4 (a) and (b)) front-end configurations are traditional homodyne (using original transmitter CW laser as LO) and DSP-assisted intradyne (using free-running $200-\mathrm{kHz}$ linewidth discrete mode semiconductor laser (DML) [23] with polarization maintaining (PM) fiber pigtail) setups. The third receiver front-end, Fig. 4(c), is similar to the traditional homodyne configuration (Fig. 4(a)) - the only difference is that the original CW transmitter laser is used for OIL of the slave laser (isolator-free DML biased at $146 \mathrm{~mA}$ ) that is subsequently used as the LO. This receiver setup allowed us to study the minimum system performance degradation due to the OIL process, as 'clean carrier' (without any modulation) is used for OIL-based carrier recovery. In all our OIL experiments the polarization of the injected light was manually aligned using a polarization controller (as shown in Fig. 4). In a practical system, a polarization tracker would be needed.
The setup shown in Fig. 4 (d) represents our receiver without the electronic PLL and was used to study the influence of the guardband width on system performance [18]. Here, $5 \%$ of the data signal is tapped off and used in our carrier recovery module, which consisted of a polarization controller (PC), variable optical attenuator (VOA), a polarization-maintaining optical circulator, and an isolator-free DML [23] that serves as the receiver LO. The principal difference between Fig. 4 (c) and (d) is that in Fig. 4 (c), only CW light is injected into the slave laser, while in Fig. 4 (d), the signal injected into the slave laser contains both the carrier tone and the data signal (thus representing a true carrier-recovery process).

Our fully-featured phase-locked-loop-assisted OIL is shown in Fig. 4 (e) (the optical part is almost-identical to that in Fig. 4 (d)). The error signal for the PLL is generated using a pilot tone that is inserted into the guardband. The error signal for the PLL was extracted from the recovered carrier (that was tapped via a 95/5 coupler and detected with a 1-GHz bandwidth photodiode). The detector output, shown in Equation (2), contains $\Delta \phi$ and is used as the error signal. However, it is carried out at the pilot tone frequency $\omega_{\mathrm{p}}$, requiring down-conversion to the base band before it could be used as the error signal. At the same time, it is relatively weak as the pilot tone which, being outside the locking range, is attenuated by the OIL process. Thus, after photodetection, we first pre-filter the beat signal with a 50-MHz bandwidth band pass filter and amplify it up to $-7 \mathrm{dBm}$ prior to down-conversion to the base band via RF mixing (Fig. 4 (e)). This error signal is then used in the slow electronic feedback loop to assist the OIL (thereby forming an OIPLL). The feedback loop consists of a $1 \mathrm{kHz}$ low-pass filter (limiting the feedback bandwidth to $<1 \mathrm{kHz}$ ) and a proportional-integral (PI) controller, New Focus LB1005.

In all our experiments with the PLL-assisted OIL, we used a $750-\mathrm{MHz}$ wide guardband (corresponding to 20 sub-channels). To get the pilot tone sidebands well within the guardband, we used a pilot tone frequency of $250 \mathrm{MHz}$. As mentioned earlier, our PLL requires the pilot tone sidebands to lie outside the OIL bandwidth. We found experimentally that this condition is met for a $250-\mathrm{MHz}$ pilot tone frequency for injection ratios below $-51 \mathrm{~dB}$. The residual pilot signal was removed in the offline processing before the FFT.

\section{EXPERIMENTAL RESULTS}

We measured the average error vector magnitude (EVM) of our QPSK-OFDM signal using various receiver front-ends (Figs. 4(a)-(d)) and show the results in Fig. 5. First, we performed homodyne detection (setup Fig. 4 (a)) obtaining an EVM of $10.7 \%$ (black dot curve). Intradyne detection (setup Fig. 4 (b)) gave an EVM of 15.1\% (green dashed curve), being by 4 percentage points worse as compared to the homodyne one. This penalty is partly due to the residual CFO and partly due to the limited linewidth $(200 \mathrm{kHz})$ of the DML used in the intradyne detection. Next, we characterized homodyne performance in which the transmitter laser was not used directly as the LO, but was first optically-injection-locked into the slave laser which served as the LO, Fig. 4 (c). The resulting EVM value at different injection ratios is plotted as closed 
squares. When the injection ratio was relatively high (above -50 $\mathrm{dB})$, the performance of the demodulated signal approached that of traditional homodyne detection (Fig. 4 (a)), indicating that the OIL can recover the carrier with high fidelity, more specifically when injection ratio is greater than $-50 \mathrm{~dB}$. Lowering the injection ratio, the EVMs of the demodulated signal increases up to a value of $13 \%$, obtained for the minimum injection ratio for which we were able to achieve a phase lock of $-74 \mathrm{~dB}$. This is because the tracking capability of the OIL process is reduced when the injected power is decreased, resulting in larger phase noise between the locked slave and the master signal [21]. This effect sets the limitation in the performance of the OIL-based carrier recovery scheme. Nevertheless, even at the lowest injection ratio obtainable (-74 $\mathrm{dB}$ ), this scheme gives an EVM about two percentile points lower than the intradyne detection.
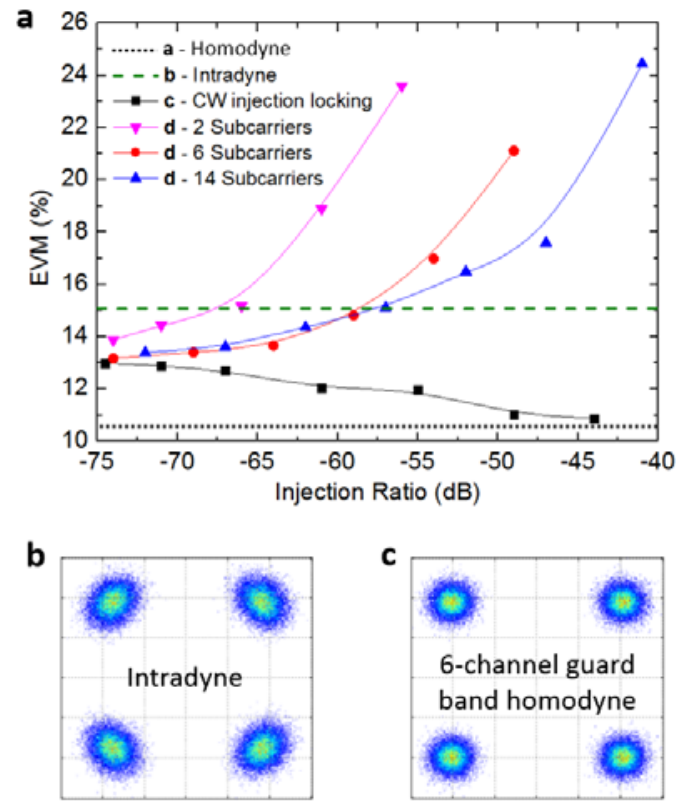

Fig. 5. a) EVM as a function of the injection ratio. Homodyne (dotted) and intradyne (dashed) detection schemes do not use OIL and thus are shown as straight lines. Squares: Clean CW beam used for OIL. Our new scheme using a guardband occupying 2 (inverse triangles), 6 (circles), and 14 (triangles) sub-channels. b) Constellation diagram for intradyne and (c) 6-channel guardband homodyne detection.
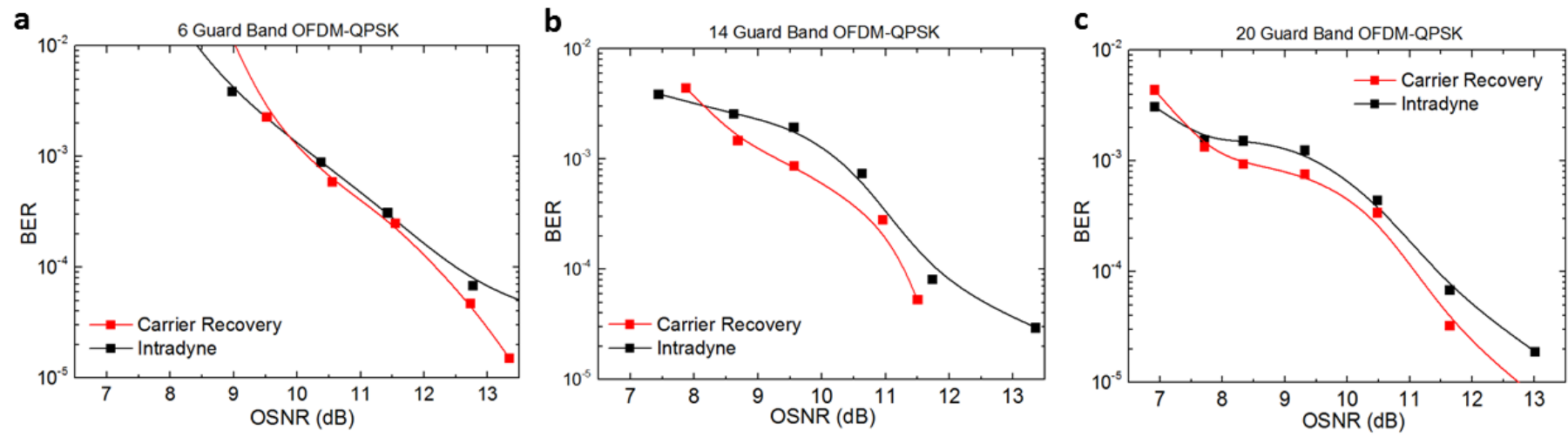

Fig. 7. BER performance of the heterodyne OFDM and carrier recovery based OFDM with different number (a) 6, (b) 14, (c) 20-subcarrier wide guard band and QPSK constellation mapping. An OIL ratio of $-70 \mathrm{~dB}$ was used. Points were connected by spline interpolation. 
guardbands at a BER of $10^{-3}$, respectively. The performance of the carrier recovered homodyne OFDM with a 6 subcarrier wide guardband is comparable to that obtained with the intradyne detection in the OSNR range from $9.5 \mathrm{~dB}$ to $12 \mathrm{~dB}$ and only showed better performance at higher OSNR. This suggests that the suppression of the low frequency subcarriers for the recovered carrier is insufficient, which counteracts the benefit provided by our OIL-based homodyne detection. As a result, a greater than 6 sub-carrier guardband width should be used for optimum performance. As BER is here affected by several phenomena (ASE, phase noise due to OIL, and insufficient signal suppression), the BER-OSNR characteristics in Fig. 7 do not follow a straight line, as would be the case if just ASE was limiting the performance.

At very low injection ratios, the OIL tends to unlock frequently. As the locking range becomes very small even a small drift of the slave or transmitter lasers' temperature or drive current causes unlocking. To ensure long-term stability, we used (i) a larger guardband (occupying 20 sub-carriers) allowing for a larger OIL bandwidth, and (ii) our phase-locked loop (setup shown in Fig. 4 (e)). With this in place the stability of our system improved substantially (allowing continuous operation over hours as compared to the previous version that went out of lock - depending on the injected power - after tens of seconds (for very low injected powers) to tens of minutes (for relatively high injected powers), allowing us to investigate more complicated modulation formats. In what follows, we use OFDM-16QAM. Once the OIL long-term stability was improved, our system became limited by the drift of the transmitter laser and this necessitated fine tuning of the 0.12-nm bandwidth filter used at the receiver. To avoid this, we replaced this filter with a $0.8-\mathrm{nm} 3-\mathrm{dB}$ bandwidth filter. This filter was also used in the intradyne detection scheme used for comparison.

To characterize the impact of the pilot tone in the recovered LO, signals with various carrier-to-pilot tone power ratios (CPR, measured in the recovered carrier) were used. Fig. 8 was obtained using a single-polarization OSNR of $34 \mathrm{~dB}$. The black squares are the EVM of the demodulated OFDM-16QAM as a function of the CPR. The dashed green line is the EVM for intradyne detection while the solid blue line is obtained using OIL-based carrier recovery without a pilot tone (Fig. 4(d)). As shown in the constellation diagrams in Fig. 8, a smaller CPR (bigger pilot power) leads to larger inter-channel interference (ICI) in OFDM demodulation, which results in worse EVM performance. The carrier-recovered homodyne OFDM outperforms intradyne detection when the CPR is larger than 27 $\mathrm{dB}$ and its performance approaches that of the carrier recovery not assisted by any pilot tone (Fig. 4 (d)) when the CPR is larger than $45 \mathrm{~dB}$. Therefore we can conclude that the pilot tone induced penalty is negligible only for $\mathrm{CPR}>45 \mathrm{~dB}$. An example is shown in Fig. 9, where the $\mathrm{CPR}=45 \mathrm{~dB}$ and the injection ratio was $-60 \mathrm{~dB}$. As expected, the recovered carrier is free of data modulation. Analyzing the original data signal (Fig. 9 (a)) we see that a CPR of $45 \mathrm{~dB}$ was obtained when the carrier-tone-to-pilot-tone ratio in the original data signal was $-18 \mathrm{~dB}$.

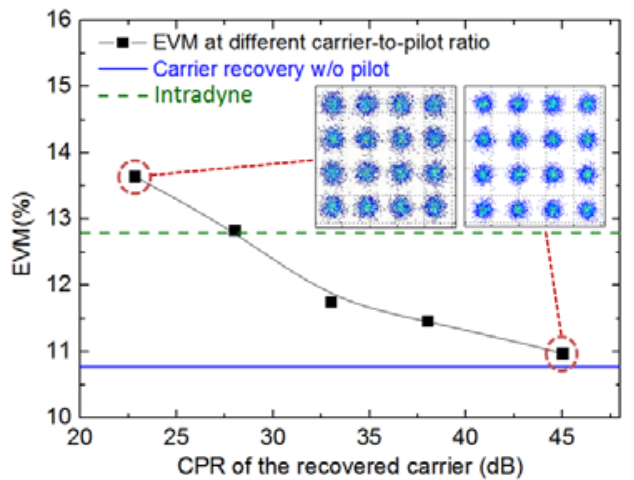

Fig. 8. EVM of the demodulated OFDM-16QAM at different CPR values; OSNR $=34 \mathrm{~dB}$.
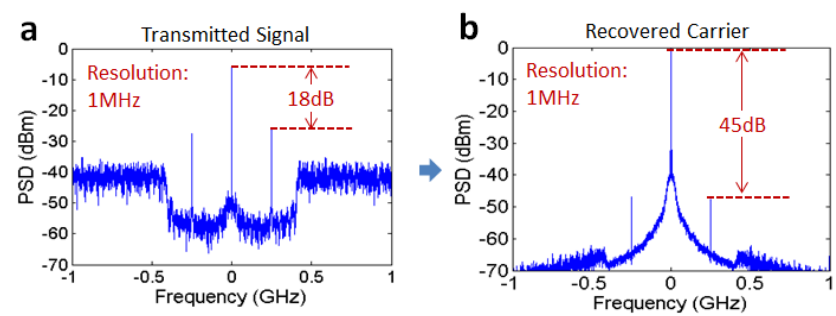

Fig. 9. Spectra of (a) the OFDM signal with a carrier-to-tone ratio of $18 \mathrm{~dB}$; (b) recovered carrier that has a CPR of $45 \mathrm{~dB}$.

The BER result of the carrier recovered OFDM-16QAM using the CPR of $45 \mathrm{~dB}$ is shown as a function of the single-polarization OSNR in Fig. 10. It can be seen that the carrier recovery scheme outperforms the intradyne approach in the high OSNR regime (BER below $10^{-4}$ ) and is slightly inferior to intradyne at low OSNR. Compared with the previous result obtained with OFDM-QPSK, we observed two phenomena: 1) the carrier recovery scheme requires a higher OSNR to outperform intradyne for OFDM-16QAM; 2) the BER results show a linear relationship with OSNR, suggesting ASE is the dominant source of noise. This is consistent with the fact we used a 0.8-nm filter, which is about 6 times wider than the bandwidth of the signal.

\section{DISCUSSION}

In the above-shown results, it seems that our homodyne scheme provides only modest performance improvements as compared to the DSP-assisted intradyne approach. The BER characteristics (Fig. 7 and Fig. 10) show comparable performance for both schemes, although for QPSK-OFDM, there is about $1 \mathrm{~dB}$ OSNR advantage of our scheme as compared to the intradyne one. However, as discussed in the introduction, there are distinct advantages of using homodyne detection. Firstly, it is computation power required for DSP. The savings depend on the system parameters (e.g., total dispersion, noise levels, etc.) and thus are difficult-to-quantify. However, to give at least a rough idea of potential savings, we used in our experiment the same computing resources for homodyne as well as intradyne detection and found that our optical carrier recovery homodyne scheme needed 1.75 times less time to process the data than the intradyne detection. This is mainly due to the savings in the CFO estimation and compensation in our offline processing. For real-time signal 
processing, our scheme would thus require a 1.75 times slower (i.e., cheaper and less power-hungry) DSP processor. Another important advantage of homodyne detection is that it requires the minimum photodetection bandwidth. This is particularly important for OFDM which is sensitive to high frequency distortion e.g., when a limited bandwidth photodiode is used. Such a penalty may occur in intradyne detection when the signal bandwidth is close to the bandwidth of the photodiode used. Such a limitation that is most likely to happen in a real system was not the case in our laboratory setup. In our experiment, we were limited by the sampling performance of our AWG rather than by photodiodes that were of very high bandwidth of $32 \mathrm{GHz}$. To get an estimate of how this effect would degrade a real system, we estimated the photodiode bandwidth-induced penalty through simulations. The parameters in the simulations are the same as in the experiment except a $20 \mathrm{GS} / \mathrm{s}$ AWG sampling rate that was used in simulations for OFDM signal of $10 \mathrm{GHz}$ base band bandwidth. The spectral shape of the photodiode was considered to have a 10-GHz 3-dB bandwidth 5th-order Butterworth shape. Due to this limited bandwidth, the high frequency subcarriers are attenuated in the photodiode. As shown in Fig. 11, a CFO of 1.5 $\mathrm{GHz}$ causes 1-dB OSNR penalty at BER of $10^{-3}$ and this penalty would increase dramatically for larger CFOs. This kind of penalty is inherently eliminated in homodyne detection.

Our experiments were carried out using a system limited to 10 GBauds (mainly due to our AWG). The largest guardband we used (setting 20 sub-channels to zero) led to an $8 \%$ reduction of the capacity. However, considering higher baud rates (e.g., 28 or 56 GBauds), the reduction of the transmission capacity would be lower (e.g., about $1 \%$ for 56 GBauds). Alternatively, we could use a larger guardband (keeping the transmission capacity reduced by $8 \%$ ). This would allow for higher injection ratios that - according to the results shown in Fig. 5 (black square dots) - should lead to lower EVM (reaching that of an ideal homodyne receiver) and thus also better performance.

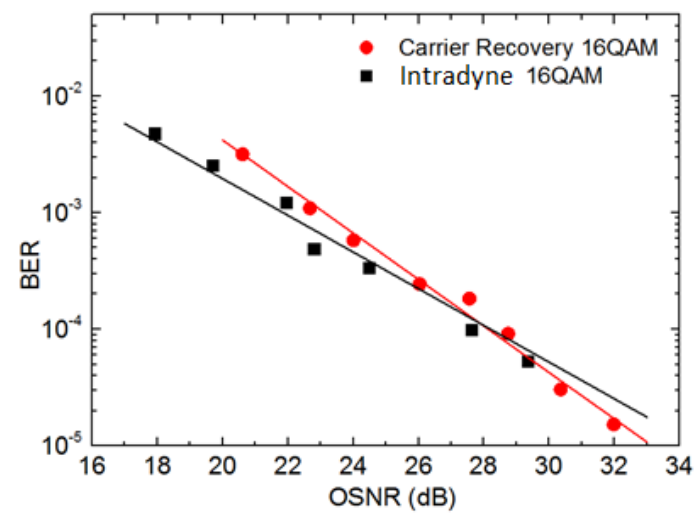

Fig. 10. BER performance of the intradyne and carrier recovery based OFDM-16QAM.

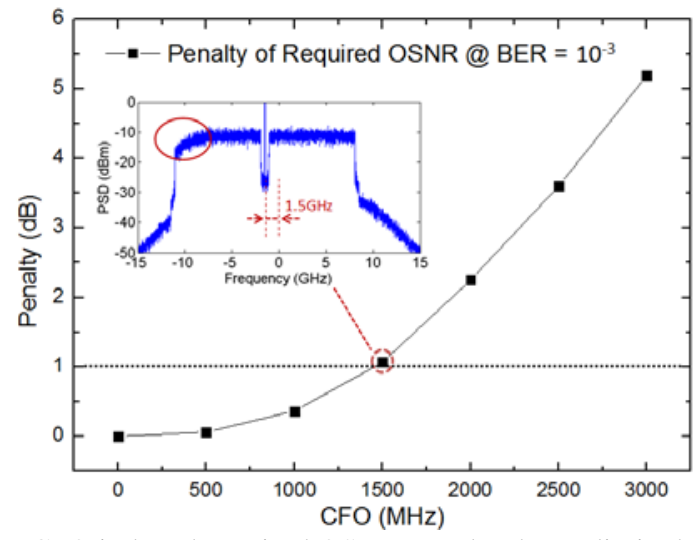

Fig. 11. CFO induced required OSNR penalty due to limited receiver bandwidth.

\section{CONCLUSION}

An OIL-based optical carrier recovery that does not require any optical pre-filtering is studied and characterized for homodyne detection of the carrier-aided OFDM. Compared to conventional intradyne detection, our carrier recovery generates a narrow linewidth LO cost-effectively and avoids the penalty induced by the residual CFO. Further, better BER performance was achieved (e.g., $1 \mathrm{~dB}$ lower OSNR penalty for QPSK-QAM at a BER of $10^{-3}$ ) simultaneously with 1.75times reduction in the computation power required for the DSP. Considering CFO drift of 1 to $1.5 \mathrm{GHz}$ in the intradyne detection, our scheme is expected to give a further $1 \mathrm{~dB}$ lower OSNR penalty, giving up to $2 \mathrm{~dB}$ lower OSNR penalty as compared to intradyne detection. Although our method requires a guardband occupying 14-20 sub-carriers, it is associated only with a relatively low reduction in the capacity by $5-8 \%$. This number will be lower if a higher baud rate would have been used (e.g., $1 \%$ at 56 GBauds). For stable and practical operation, we implemented a slow $(<1 \mathrm{kHz})$ phase locked loop, enabling reliable transmission of OFDM-16QAM with negligible impact on the system performance.

\section{REFERENCES}

[1] J. Amstrong, “OFDM for Optical Communications," IEEE J. Lightw. Technol., vol. 27, no. 3, pp. 189-204, Feb. 2009.

[2] W. Shieh, "OFDM for Flexible High-Speed Optical Networks," IEEE J. Lightw. Technol., vol. 29, no. 10, pp. 1560-1577, May. 2011.

[3] W. Shieh, I. Djordjevic, "Chapter 2: OFDM Principles," in Orthogonal frequency division multiplexing for optical communications, Academic Press, 2010.

[4] B. J. Puttnam, R. S. Luís, J. M. D. Mendinueta, J. S.akaguchi, W. Klaus, Y. Kamio, M. Nakamura, N. Wada, Y. Awaji, A. Kanno, T. Kawanishi and T. Miyazaki, "Self-Homodyne Detection in Optical Communication Systems,” Photonics, vol. 1, no. 2, pp. 110-130, May, 2014.

[5] A. Barbieri, G. Colavolpe, T. Foggi, E. Forestieri, and G. Prati, "OFDM versus Single-Carrier Transmission for 100 Gbps Optical Communication," IEEE J. Lightw. Technol., vol. 28, no. 17, pp. 2537-2551, Sept., 2010.

[6] X. Zhou, X. Yang, R. Li, and K. Long, "Efficient Joint Carrier Frequency Offset and Phase Noise Compensation Scheme for High-Speed Coherent Optical OFDM Systems," IEEE J. Lightw. Technol., vol. 31, no. 11, pp. 1755-1761, June, 2013.

[7] A. Mizutori, S. Y. Set, F. Shirazawa, M. Koga, "Stable Costas loop homodyne detection for 20-Gbit/s QPSK signal fiber transmission," in Proc. Eur. Conf. Optical Commun. (ECOC), London, UK, Sept. 2013, Paper Mo.4.C.1. 
[8] Y. Wang, K. Kasai, T. Omiya, and M. Nakazawa, "120 Gbit/s, polarization-multiplexed 10Gsymbol/s, 64 QAM coherent transmission over $150 \mathrm{~km}$ using an optical voltage controlled oscillator,” Opt. Express, vol. 21, no. 23, pp. 28290-28296, Nov., 2013.

[9] Z. Liu, J. Kakande, B. Kelly, J. O’Carroll, R. Phelan, D. J. Richardson, and R. Slavík, "Coherent Optical OFDM based on Direct Modulation of Injection-locked Fabry-Perot Lasers," in Proc. Eur. Conf. Optical Commun. (ECOC), Cannes, France, Sept. 2013, Paper Tu.3.6.3.

[10] S. L. Jansen, I. Morita, T. C. W. Schenk, N. Takeda, and H. Tanaka, "Coherent Optical 25.8-Gb/s OFDM Transmission Over 4160-km SSMF”, IEEE J. Lightw. Technol., vol. 26, no. 1, pp. 6-15, Jan., 2008.

[11] B. Inan, S. Randel, S. L. Jansen, A. Lobato, S. Adhikari, N. Hanik, "Pilot-Tone-based Nonlinearity Compensation for Optical OFDM Systems", in Proc. Eur. Conf. Optical Commun. (ECOC), Turin, Italy, Sept. 2010, Paper Tu.4.A.6.

[12] S. Adhikari, S. Sygletos, A. D. Ellis, B. Inan, S. L. Jansen, and W. Rosenkranz, "Enhanced Self-Coherent OFDM by the Use of Injection Locked Laser," in Proc. Opt. Fiber Commun. Conf. (OFC), 2012, paper JW2A.64.

[13] M. J. Fice, A. Chiuchiarelli, E. Ciaramella, and A. J. Seeds, "Homodyne Coherent Optical Receiver Using an Optical Injection Phase-Lock Loop," IEEE J. Lightw. Technol., vol. 29, no. 8, pp. 1152-1164, Apr., 2011.

[14] A. Chiuchiarelli, M. J. Fice, E. Ciaramella, and A. J. Seeds, "Effective homodyne optical phase locking to PSK signal by means of 8b10b line coding," Opt. Express, vol. 19, no. 3, pp. 1707-1712, Jan., 2011.

[15] A. Bogris, C. Ressopoulos, "Analysis of 50 GBaud homodyne coherent receivers relying on line-coding and injection locking in lasers," in Proc. Conf. on Lasers and Electro-Optics (CLEO), 2014, paper JTh2A.

[16] R. S. Luís, B. J. Puttnam, J. D. Mendinueta, S. Shinada and N. Wada, "Self-homodyne CO-OFDM packet transmitter with polarization-multiplexed pilot tone," in Proc. OptoElectronics and Communications Conference held jointly with 2013 International Conference on Photonics in Switching (OECC/PS), 2013, paper TuT3-2.

[17] R. S. Luis, B. J. Puttnam, J. D. Mendinueta, J. Sakaguchi, S. Shinada, Y. Kamio, N. Wada, and M. Nakamuraand, "Self-homodyne coherent OFDM packet transmission without carrier frequency or common phase error estimation,” IEEE 4th International Conference on Photonics (ICP), pp. 123-125, 2013.

[18] Z. Liu, D. S. Wu, D. J. Richardson, and R. Slavík, "Homodyne OFDM using Simple Optical Carrier Recovery," in Proc. Opt. Fiber Commun. Conf. (OFC), 2014, paper W4K.3.

[19] A. E. Siegman, "Chapter 29: Laser Injection Locking" in Lasers, University Science Books, 1986.

[20] F. Mogensen, H. Olesen, and G. Jacobsen, "Locking Conditions and Stability Properties for a Semiconductor Laser with External Light Injection,” IEEE J. Quantum Electron., vol. QE-21, no. 7, pp.784-793, Jul., 1985.

[21] D. S. Wu, R. Slavík, G. Marra, D. J. Richardson, "Direct Selection and Amplification of Individual Narrowly Spaced Optical Comb Modes via Injection Locking: Design and Characterization”, IEEE J. Lightw. Technol., vol. 31, no. 14, pp. 2287-2295, Jul,, 2013.

[22] R. Slavík, J. Kakande, and D. J. Richardson, "Feed-forward true carrier extraction of high baud rate phase shift keyed signals using photonic modulation stripping and low-bandwidth electronics," Opt. Express, vol. 19, no. 27, pp. 26594-26599, Dec. 2011.

[23] J. O'Carroll, R. Phelan, B. Kelly, D. Byrne, L. P. Barry, and J. O’Gorman, "Wide temperature range $0<\mathrm{T}<85^{\circ} \mathrm{C}$ narrow linewidth discrete mode laser diodes for coherent communications applications”, Opt. Express, vol. 19, no. 26, pp. B90-B95, 2011.

Zhixin Liu (S'07-M'12) received the B.Eng. degree in information engineering and B.B.A. degree in business administration from Tianjin University, China in 2006, M.S. degree in electrical engineering from Shanghai Jiao Tong University, China, in 2009, and Ph.D. degree in information engineering from the Chinese University of Hong Kong, China, in 2012.

In 2009, he worked as a qualification engineer in Corning Shanghai Fiber Optics Co., Ltd.. From 2013 he is with Optoelectronics Research Centre (ORC), University of Southampton, Southampton, U.K. His research interest includes the digital/optical subsystem, transceivers, and optical metro/access network.

Joon-Young Kim received the Ph.D. degree in electrical engineering from Korea Advanced Institute of Science and Technology (KAIST), Daejeon, Korea in 2013.

He joined the Optoelectronics Research Centre (ORC) in University of Southampton as a research fellow. Currently, his research interest includes the field of optical comb and ultra-stable optical frequency transfer over optical fibre network.

David S.Wu received the B.Sc. (advanced) (Hons.) degree in physics from the University of Sydney, Australia in 2009 and is currently pursuing the Ph.D. degree at the Optoelectronics Research Centre at the University of Southampton, U.K.

He has held a summer vacation scholarship at Australia's Commonwealth Scientific and Industrial Research Organisation (CSIRO). His research interestsinclude optical injection locking, optical frequency combs, microstructured optical fibers and optical metrology.

D. Wu is a student member of OSA and SPIE.

David J. Richardson (M.I.E.E.E.) obtained B.Sc. and Ph.D. in fundamental physics from Sussex University U.K. in 1985 and 1989 respectively.

He joined the Optoelectronics Research Centre (ORC) at Southampton University in 1989 and was awarded a Royal Society University Fellowship in 1991 in recognition of his pioneering work on short pulsed fiber lasers. Professor Richardson is now a Deputy Director of the ORC with responsibility for the ORC's fiber related activities. His current research interests include amongst others: optical fiber communications, microstructured optical fibers and pulsed high-power fibre lasers. He is a prominent figure in the international photonics community and has published more than 900 conference and journal papers and produced more than 20 patents.

Professor Richardson is a Fellow of the Optical Society of America, the Institute of Engineering and Technology and was made a Fellow of the Royal Academy of Engineering in 2009.

Radan Slavík (M’07-SM’07) received the M.A.Sc. and Ph.D. degrees in optics and optoelectronics from the Faculty of Mathematics and Physics, Charles University in Prague, in 1996 and 2000, respectively. He received D.Sc. degree from Academy of Sciences of the Czech Republic in 2009.

In 1995-2000 and 2004-2009 he was with the Institute of Photonics and Electronics, Czech Academy of Sciences in Prague. In 2000-2003, he was with the Centre d'optique, photonique et laser at Université Laval, Québec, Canada as a Postdoctoral Research Fellow. From 2009 he is with Optoelectronics Research Centre (ORC), University of Southampton, Southampton, U.K. His research interests focus in optical and optics-assisted signal processing.

R. Slavík is a member of OSA. In 2006, he received Otto Wichterle Award and in 2007, he received The Visegrad Group Academies Young Researcher Award. 